\title{
LOS ESTUDIANTES UNIVERSITARIOS ANTE LA CIENCIA
}

GUADALUPE RUIZ CUÉLLAR

Departamento de Educación/UAA

Victoria Eugenia Gutiérrez Marfileño, Actitudes de los estudiantes hacia la ciencia, Aguascalientes, Programa Interinstitucional de Investigaciones sobre Educación Superior/ Universidad Autónoma de Aguascalientes, 1998.

Al libro que reseñamos es el producto de un trabajo de investigación emprendido por la autora para obtener el grado de Maestra en Educación. Como ella lo señala, más que limitarse a la presentación de esa investigación, el libro tiene una intención didáctica: pretende mostrar clara y sistemáticamente todo el proceso seguido para desarrollar el estudio. Se trata de un trabajo a medio camino entre el informe técnico y el libro; no es tan áspero como podría serlo casi por necesidad un informe, pero tampoco es una obra que se des- prenda de los referentes que aportan los protocolos de investigación. Estos, normalmente reflejan la lógica del quehacer investigativo, que no necesariamente es la misma lógica que debe seguir la exposición de un estudio. Construir una lógica discursiva - cosa de la que creo que es perfectamente capaz Victoria Eugeniano se echa de menos en el libro porque, a cambio de ella tenemos un excelente reporte de investigación que cumple, a mi modo de ver, con la pretensión didáctica de la autora. Desconozco si ella misma o a sugerencia de otros decidió que el público al que de manera preferente se destinase la obra fuese el formado por "aquéllos que empiezan a incursionar en el terreno de la investigación educativa". En cualquier caso, creo que contamos con un valioso material de apoyo para las tareas de formación metodológica para la investigación que realizamos en el Departamento 
de Educación de la UAA, e incluso en otras instituciones.

Esta preocupación se evidencia en el desarrollo minucioso que hace de varios apartados: por ejemplo, la revisión de la literatura, donde, no se limita a referir ciertos aspectos de los trabajos previos identificados, sino que ofrece un panorama amplio sobre éstos, aplicando a cada uno un esquema común de análisis que le lleva a mostrar, entre otras cosas, las preocupaciones que motivan al estudio de las actitudes hacia la ciencia, los enfoques teóricos y las disciplinas a la base de dichos estudios, los conceptos de actitud manejados, los acercamientos metodológicos, etc. 0 bien, lo relativo al diseño metodológico, donde queda particularmente de manifiesto cuál es la lógica seguida por la autora para construir el instrumento a través del cual obtuvo la información necesaria para el estudio. Por supuesto, este afán didáctico se aprecia también en la exposición de los resultados.

\section{LA INVESTIGACIÓN SOBRE ACTITUDES HACIA LA CIENCIA}

¿Por qué estudiar las actitudes? El tema de las actitudes ha atraído el interés de varios egresados de los programas del Departamento de Educación orientados a la formación para la investigación educati- va; podemos encontrar tesis de licenciatura y maestría que varían en cuanto al objeto de actitud que abordan, pero coincidentes en el planteamiento básico, esto es, el acercamiento a las actitudes como una variable potencialmente poderosa para explicarnos un sinnúmero de fenómenos educativos y sociales.

Los educadores, preocupados como es lógico por la calidad de la educación, atendemos con interés particular los indicadores de la medida en que el sistema educativo en los diferentes niveles que lo conforman logra sus propósitos. Es decir, nos interesa su eficacia, concebida como dimensión sustantiva de la calidad.

En cualquier país del mundo, la educación primaria, por ejemplo, sirve a varios propósitos. Uno de ellos es favorecer el desarrollo de habilidades cognitivas básicas por parte de los estudiantes; un segundo propósito es desarrollar actitudes y habilidades que los niños necesitan para funcionar efectivamente en sociedad. Uno más, el avanzar en el desarrollo nacional. Si atendemos al contexto concreto de nuestro estado, la Ley de Educación establece en su artículo 12 que "... la educación que se imparta, promueva y atienda en el Estado de Aguascalientes perseguirá los siguientes fines (cito sólo algunos y no en todos los casos en toda su extensión): 
- contribuir al desarrollo integral del individuo para que ejerza plenamente sus capacidades humanas;

- favorecer el desarrollo de facultades para adquirir valores, conocimientos y habilidades, así como la capacidad de observación, análisis y reflexión críticos;

- fortalecer la conciencia de la nacionalidad y de la soberanía ...;

- infundir el conocimiento y la práctica de la democracia ...;

- promover el valor de la justicia, de la observancia de la ley y de la igualdad de los individuos ante ésta ...;

- fomentar actitudes que estimulen la investigación y las innovaciones científicas y tecnologicas;

- impulsar la creación artística ...;

- hacer conciencia de la necesidad de un aprovechamiento racional de los recursos naturales y de la protección del ambiente ..."

Aunque, como se puede ver, los propósitos expresan muchas áreas de desarrollo de los individuos sobre las que nuestro propio sistema educativo estatal -pero en realidad, prácticamente cualquiera- pretende incidir, lo cierto es que en muchos casos, tiende a privilegiarse el propósito de desarrollar competencias cognitivas en los educandos. Parece haber, de hecho, una menor preocupación traducida en acciones concretas y menos herramientas quizás, para atender su formación valoral y actitudinal.

Desde una perspectiva investigativa, las actitudes resultan ser una variable que bien puede ser vista como antecedente, como consecuencia de otras, o bien, esta- bleciendo con ellas una relación bidireccional de influencia recíproca; en cualquier caso, su importancia es capital. Las actitudes hacia determinadas materias o actividades humanas (la ciencia y las ciencias en el caso de este trabajo particular), se asocian a otras variables de tipo afectivo, cognitivo y conductual y parecen tener un peso considerable en la explicación del éxito o fracaso escolar, de trayectorias vocacionales, profesionales y ocupacionales, etc.

De ahí que el definirlas como objeto de estudio sea sin duda un imperativo fundamental. En este sentido, es motivo de celebración contar con la obra de Victoria Eugenia Gutiérrez Marfileño. La investigación elige como su ámbito empírico de indagación el nivel universitario. Si, como el estudio trata de mostrar, apoyado en la vasta evidencia arrojada por otras investigaciones, las actitudes se gestan a lo largo de periodos de tiempo prolongados, lo que encontramos que caracteriza a los universitarios del presente, debe remitirnos a su trayectoria escolar pasada, a lo que hace y deja de hacer la escuela en los niveles educativos previos. En este sentido, los resultados que arroja el estudio trascienden el interés por el tema en el marco del nivel de educación superior y deberían ser materia de reflexión y preocupación de los educadores de todos los niveles del sistema educativo. 


\section{EDUCACIÓN CIENTIFICA E INVESTIGACIÓN}

A manera de contextualización de su propia investigación, la autora destaca claramente el papel central que ocupa la ciencia en la sociedad moderna; en pocas palabras -dice ella, citando a algunos autores- "el mundo lleva el sello de la ciencia". Reconociendo este hecho, la escuela asume la educación científica como un asunto de carácter prioritario. La educación científica, dice la autora,

...no sólo incluye enseñar y aprender ciencia, como una actividad humana que se caracteriza por la producción de un tipo especial de conocimiento ... la educación científica pretende formar una disposición favorable hacia la ciencia como actividad y como producto; formar una disposición favorable hacia aquéllos que la realizan, y en algunos casos, llevar esa buena disposición hasta el deseo de ser parte dinámica de dicha actividad. Incluiría además, valorar la ciencia como un rasgo importante de nuestra sociedad, una actividad y un producto que han llevado al conocimiento del mundo; un conocimiento, que si bien ha tenido efectos secundarios indeseables, no por ello, debe ser eliminado. Se trata, entonces, de que educando a las personas en la ciencia y para la ciencia, ya sea como usuarios inteligentes y críticos, o bien como productores de conocimiento, se vaya al mismo tiempo desarrollando la sensibilidad para reconocer lo adecuado en función de la sociedad y de su bienestar... Lo anterior puede lograrse si en la educación científica son atendidos tanto los aspectos cognitivos como la dimensión afectiva, particularmente la que se refiere a las actitudes y los valores (pp. 16-17).
Aunque el estudio realizado por Victoria Eugenia no es un proyecto de intervención educativa, dado que sus propósitos están puestos en la generación de conocimientos sobre un aspecto particular del campo más amplio de la educación científica, esto es, las actitudes de los estudiantes hacia la ciencia, algunos hallazgos del estudio son preocupantes e indicativos de que debería prestarse una mayor atención a la educación científica de nuestros niños y jóvenes. Destaco una parte de los que me parecen más importantes:

- Los estudiantes de la muestra -algunos de los cuales estudian carreras científicas y otros, carreras no cientificas'- "poseen poca información en cuanto a la naturaleza o características de la actividad científica; incluso puede decirse que dicha información no es del todo exacta ...". Citando a algunos estudiosos, la autora señala que las investigaciones recientes sobre la enseñanza y aprendizaje de la ciencia muestran "que sigue transmitiéndose en la escuela una visión muy deformada del trabajo cientifico ..." (pp. 145-146).

${ }^{1}$ Cabe mencionar lo que la autora señala a este respecto: "... si bien, en los planes de estudio de todas las carreras impartidas en la UAA existe un énfasis en la formación científica de los estudiantes, al interno de dicha formación se identifican dos perfiles: uno, orientado a definir al estudiante como un usuario competente de la ciencia, es decir, un profesional que fundamente su práctica en el conocimiento científico en su campo. El otro perfil atiende a la formación del profesional, como productor de ciencia, se trata entonces, de la formación de investigadores en algún campo del conocimiento". (p. 75.) 
- "En este estudio los alumnos consideraron únicamente como cientificas aquellas actividades que claramente estaban relacionadas con procedimientos experimentales ... incluso, actividades que pueden realizarse en una investigación, que en sentido estricto no son la investigación pero que hacen alusión a trabajo de laboratorio ... fueron calificadas como trabajo científico ..." (p. 146).

- Los estudiantes de esta muestra califican de no científicos trabajos cuyo objeto de estudio no es directamente observable; es el caso de los estudios en las ciencias del hombre. Coincidentemente con los resultados de otros estudios, la autora encuentra que los estudiantes consideran como ciencia únicamente a las disciplinas duras.

- Una visión velada y elitista de la ciencia es otra de las concepciones transmitida por la escuela... El trabajo científico es presentado como un dominio reservado a las minorías particularmente dotadas, transmitiendo expectativas negativas hacia la mayoría de los alumnos con claras distinciones de naturaleza social y sexual (la ciencia es vista como actividad eminentemente masculina).

- Cuando a los estudiantes se les cuestionó sobre los científicos y el trabajo que éstos realizan, manifestaron ideas estereotipadas: los científicos son los buscadores de la verdad. son muy críticos y tienen mentes privilegiadas. El trabajo que ellos realizan (y que en general fue valorado de manera muy favorable) es importante, interesante. valioso, útil. bueno; pero por otro lado es percibido como complejo, difícil y extraño.

Reitero que el trabajo de Victoria Eugenia Gutiérrez no es una propuesta de intervención educativa; no nos dice cómo favorecer mejores prácticas de educación científica. En todo caso, no es éste el propósito de su estudio. Lo que sí logra es do- cumentar para nuestro contexto específico las limitaciones de la enseñanza, que se manifiestan en visiones distorsionadas y erróneas de lo que es la ciencia como actividad y como producto; en actitudes (desde el punto de vista afectivo) no precisamente desfavorables, pero sí en prácticas de rechazo de la actividad. Las tendencias de la elección profesional, por ejemplo, muestran lo poco atractivas que resultan para la gran mayoría de los estudiantes que ingresan a la educación superior las profesiones científicas.

Quisiera insistir en la importancia capital de la educación científica. Para ello me permitiré citar con cierta amplitud algunas ideas particularmente sugerentes desde mi punto de vista: ${ }^{2}$

Desde la década de los cincuenta se ha generalizado la discusión a nivel internacional sobre la importancia de la formación científica en la educación básica. al reconocer que la ciencia y la tecnología tienen un papel muy importante en el desarrollo de las sociedades actuales. En ese contexto, durante los últimos quince años se ha planteado como uno de los retos para la educación básica incluir entre sus metas la cultura cientifica y tecnológica; consideran-

${ }^{2}$ Tomadas de una antología preparada en la Fundación SNTE para la Cultura del Maestro Mexicano -institución académica autónoma cuyo objeto es contribuir a mejorar la calidad de la educación básica-, titulada Contenidos relevantes de Ciencias Naturales para la Educación Básica, México, 1997. 
do que un ciudadano con ese tipo de cultura, es aquel que está capacitado para comprender a la ciencia y a la tecnología como procesos sociales, para comprender y valorar sus productos, hacer frente a los problemas que plantean los avances científicos y tecnologicos, y poder distinguir las aplicaciones benéficas de las que serán probablemente nocivas.

Las recomendaciones de la comunidad intemacional sobre calidad de la educación, como las emanadas de la reunión sobre Educación para todos de 1990, recogen los planteamientos sobre enseñanza de las ciencias desarrollados a nivel intemacional, acerca de la necesidad de revisar el estado actual de la calidad de la enseñanza de las ciencias en la educación básica, para adecuar los planes y programas así como las prácticas de enseñanza, tendientes al logro de la cultura científica y tecnológica para todos.

El objetivo general resulta claro: es necesario que todos los egresados del sistema de educación básica tengan una cultura cientifica, porque ésta es necesaria para entender los procesos de la sociedad actual. Sin embargo, las experiencias de las reformas curriculares desarrolladas en diferentes países, especialmente las que han tenido lugar de 1980 a la fecha y que explicitaron entre sus metas la cultura científica para todos los niños y jóvenes, demuestran que el logro de tal objetivo exige un esfuerzo considerable de todas las instancias involucradas en la planeación y operación de la educación básica en cada país. Ese proposito requiere la revisión de todos los aspectos del sistema educativo, para determinar cambios que efectivamente propicien procesos educativos orientados al desarrollo de una cultura cientifica, cambios que deben atender principalmente al currículo, la formación de los profesores, la organización y el equipamiento de los centros escolares.

Ante todo, es necesario precisar qué significa el término cultura científica para la educación básica en el contexto nacional, cuáles son los elementos concretos de tal cultura, y qué tipo de competencias requiere. A partir de esa definición, se puede construir un marco de referencia para evaluar el sistema educativo actual y determinar las modificaciones necesarias, no sólo en planes y programas, sino en todos sus componentes, como la formación y perfil de desempeño de los docentes, así como las condiciones de la institución escolar, la relación entre la escuela y la comunidad, y todos los elementos que determinan la calidad de la educación.

La importancia es manifiesta y el reto como se avizora es de grandes proporciones; formar en y para la ciencia supone empezar a actuar desde la educación básica. De no hacerlo así -como nos lo muestra el trabajo que ahora presentamos-, seguirán reproduciéndose estereotipos y visiones deformadas sobre la ciencia, el trabajo científico y los científicos.

\section{¿INVESTIGACIÓN CUANTITATIVA \\ 0 CUALITATIVA?}

No puedo resistirme a hacer un comentario final a propósito del enfoque de investigación subyacente a la obra.

Toda investigación se hace desde ciertos supuestos y fundamentos no todos los cuales necesariamente se hacen explícitos. Es el caso de los presupuestos epistemológicos del trabajo de Victoria Eugenia que, sin ser explícitos, están presentes.

Al igual que ella, creo que la mayoría 
de los maestros del Departamento de Educación de la UAA que nos dedicamos centralmente a la investigación y a la formación metodológica para esta actividad, compartimos una visión de la investigación científica que este libro muestra claramente. Actitudes de los estudiantes hacia la ciencia aborda el fenómeno bajo estudio desde una óptica predefinida, presuponiendo ciertas hipótesis y enfocando la búsqueda de información hacia ciertas variables cuya importancia deviene de los hallazgos de investigaciones previas. Es -como se diría en la jerga-, un estudio altamente estructurado, hipotético-deductivo y de enfoque cuantitativo.

Creo que algo que también nos caracteriza cuando abordamos la espinosa cuestión de los paradigmas de la investigación científica en las ciencias sociales es una actitud de respeto a la diferencia. Mucho se ha escrito -y sin duda seguirá escribiéndose- sobre este punto. Prácticamente todos los que se ocupan del tema coinciden en que, aunque se corre el riesgo de la simplificación, la enorme diversidad de enfoques particulares de investigación puede ser agrupada bajo dos paradigmas: el cuantitativo y el cualitativo, grandes vertientes que reúnen una gama heterogénea de maneras de hacer la investigación a la base de las cuales hay, sin duda, concepciones muy distintas sobre la natura- leza y la forma en que se genera el conocimiento, sobre el papel de la teoría en este proceso, sobre el papel del investigador y el de los objetos o sujetos de estudio, etc. Los grandes paradigmas: ¿opuestos 0 complementarios? ¿Conciliables o irreconciliables? ¿Explicación o interpretación? No pretendo dar una respuesta -respuesta que, por lo demás, sería una más y muy modesta en la ya añeja y erudita discusión sobre la cuestión; las polémicas, como lo documenta Felipe Martínez Rizo, se remontan al siglo XVII y con seguridad continuarán en el siglo venidero-; pretendo únicamente destacar mi convicción de que desde cualquier paradigma se puede hacer investigación de calidad e investigación deficiente; creo que -nuevamente, desde cualquier paradigma-, la investigación de calidad es aquella que aúna creatividad, objetividad, rigor y sistematicidad.

Publicada bajo el sello editorial de la UAA y del Programa Interinstitucional de Investigaciones sobre Educación Superior, Actitudes de los Estudiantes Hacia la Ciencia está disponible y al alcance de educadores, investigadores y otras personas interesadas en la temática. Convencida de que un sólido aprendizaje de la metodología de investigación en el paradigma más estructurado es lo deseable desde la perspectiva estricta de la formación para 
el quehacer investigativo, creo que contamos ahora con un excelente material de trabajo.

Sé -porque he seguido de cerca la trayectoria profesional de la autora- que su desarrollo como investigadora continúa. Probablemente esto influya para que, a cierta distancia, sea ella misma la que ejerza una mayor crítica sobre este trabajo o sobre otros que le precedieron. Eso -creo yo- es inevitable, pero saludable y alentador. Poder mirar críticamente un producto pasado o "acabado" de nuestro esfuerzo profesional, indica que seguimos creciendo, que adquirimos nuevos conocimientos y desarrollamos o adquirimos nuevas habilidades. En un terreno como el de la investigación educativa ese reto con uno mismo, es vital; espero que no pase mucho tiempo para que podamos comentar una nueva aportación de la Mtra. Victoria Eugenia Gutiérrez Marfileño al conocimiento sobre la vasta problemática educativa de nuestro tiempo. 